\title{
Catch up growth and adolescent adipokine profile in children born preterm
}

\author{
Claire Wood ${ }^{1,2}$, Murthy Korada', Kay Mann ${ }^{3}$,Tim Cheetham ${ }^{1,2}$, Nicholas Embleton ${ }^{1,3}$
}

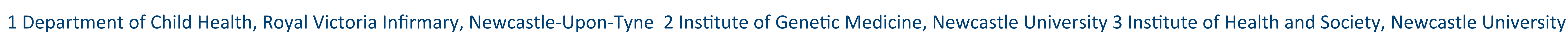

\section{Background}

Data remain conflicting regarding the consequences of prematurity and the impact of early nutrition and catch-up growth on long-term metabolic outcomes. Adiponectin and leptin are adipocyte derived proteins (adipokines) and are thought to be important regulators of insulin action.

Participants were recruited from the Newcastle Preterm Birth Growth Study (NPBGS), a prospective, randomised study of infant growth ${ }^{1}$. 102 underwent venepuncture and DEXA at a mean of 11.3 years. Z-score weight changes between term and 12 weeks were compared with adolescent adipokine levels using multivariable linear regression to adjust for potential confounders (birthweight, gestation, fat mass index, sex and pubertal status). Participants were divided into 3 subgroups depending on catch up growth: no catch up= change weight z-score $<0$, slow catch up= change $z$-score $0-0.67$, rapid catch up= change $z$-score $>0.67$. Insulin sensitivity was measured using the HOMA 2 calculator. ${ }^{2}$

\section{Results}

Overall, adipokine levels did not vary by sex. Stratification by pubertal status (Tanner stage 1 versus $>1$ ) showed significantly higher leptin levels in pubertal females than males and remained after adjustment for fat mass index. Infant growth patterns were not significantly associated with adolescent adipokine levels.

\begin{tabular}{|l|l}
\hline $\begin{array}{l}\text { Cohort } \\
\text { characteristics }\end{array}$ & Mean (range) \\
\hline $\begin{array}{l}\text { Gestational age (wks) } \\
\text { Birthweight (g) }\end{array}$ & $30.9(24.7-36.4)$ \\
\hline Birthweight z-score & $-0.91(-3.63,0.8198)$ \\
\hline Age at follow-up (yrs) & $11.3(8.7-14.2)$ \\
\hline Weight z-score & $0.24(-2.41,2.67)$ \\
\hline Height z-score & $0.00(-2.12,2.67)$ \\
\hline Insulin sensitivity & $266.1(41.1,2764.5)$ \\
\hline Adiponectin & $7.28(1.9,33.1)$ \\
\hline Leptin & $10.8(0.1,57.3)$ \\
\hline
\end{tabular}

Relationship of adiponectin to puberty and sex

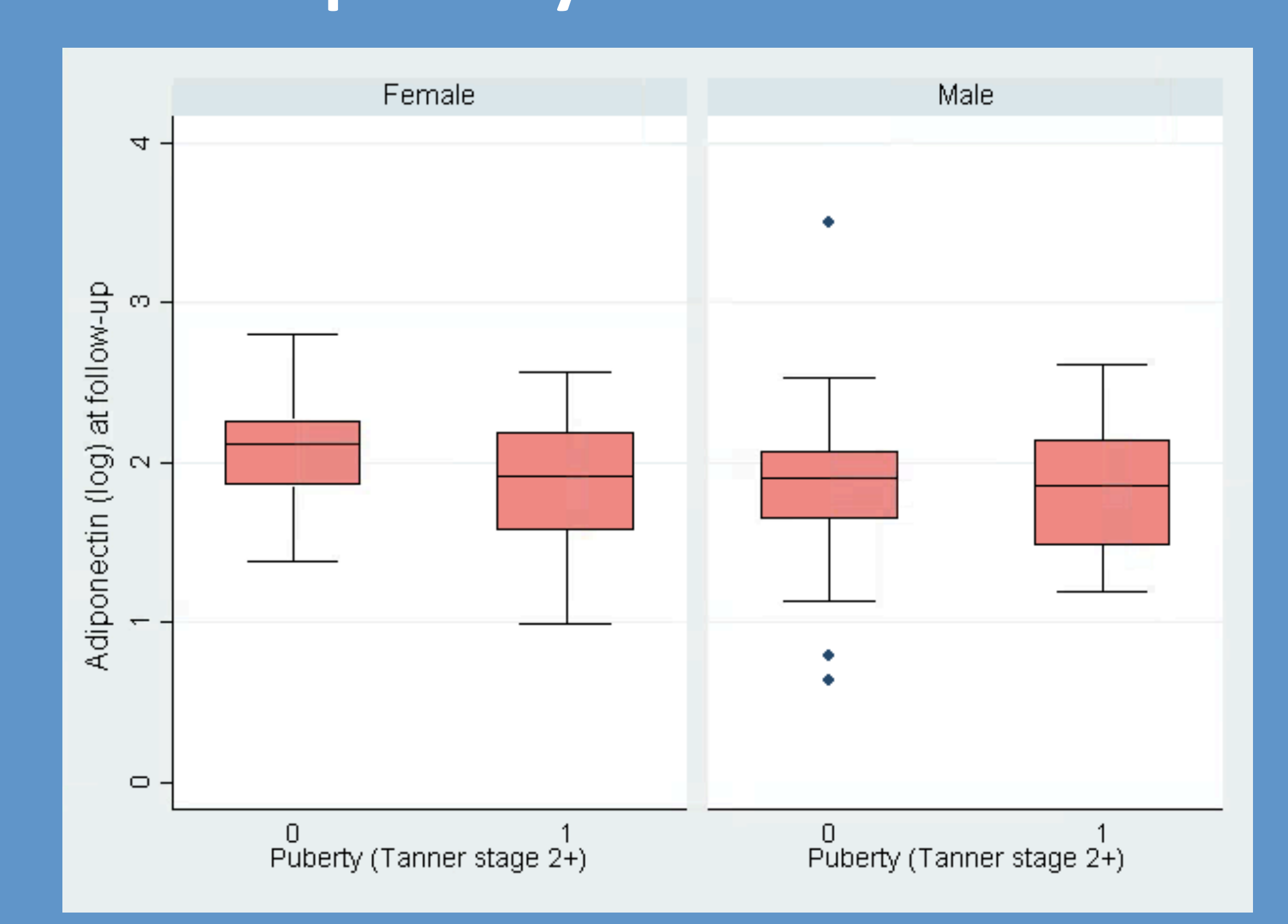

Relationship of leptin to puberty and sex

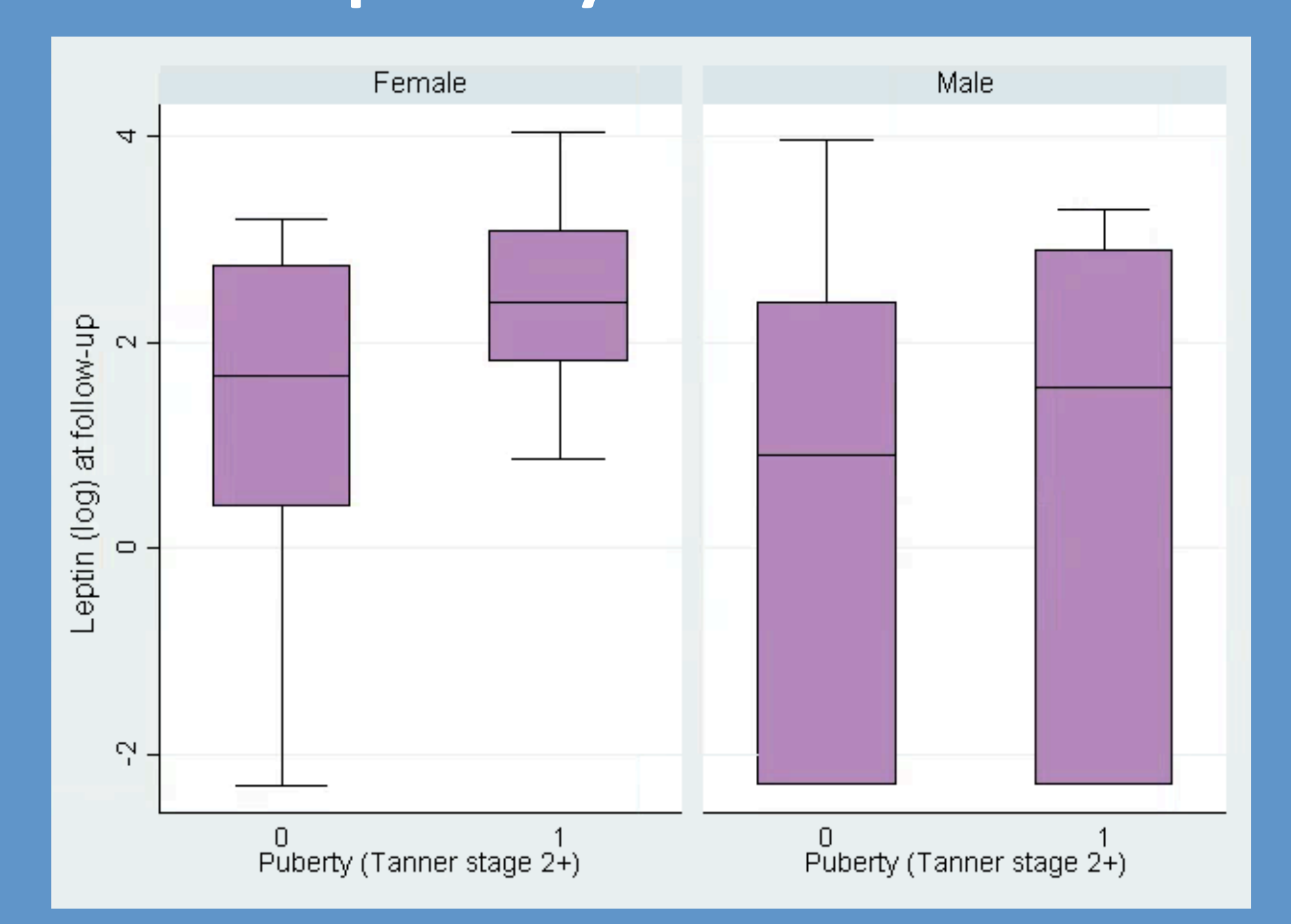

Effect of early growth on leptin at follow-up

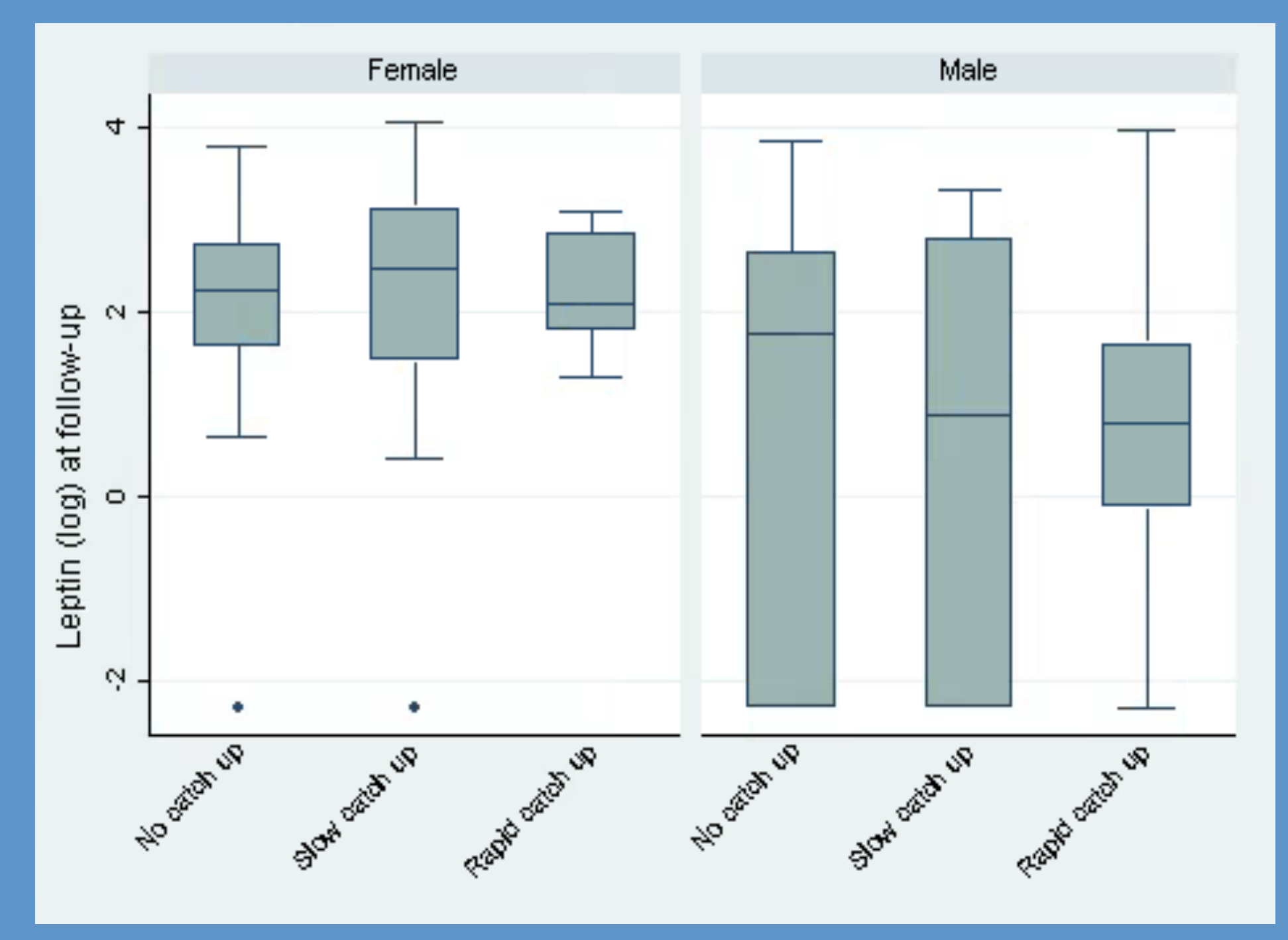

Adiponectin levels were negatively correlated with adolescent fat mass index (Spearmans correlation $=-0.25, p=0.01$ ), while leptin was positively correlated (Spearmans correlation $=0.90, p=0.00$ ); these associations remained after multivariate adjustment.

The correlations of leptin-adiponectin ratio (LAR) and leptin to insulin sensitivity (both Spearmans $=-0.58, p=0.000$ ) were equal and stronger than that of adiponectin alone. (Spearmans $=0.20, p=0.05$ )
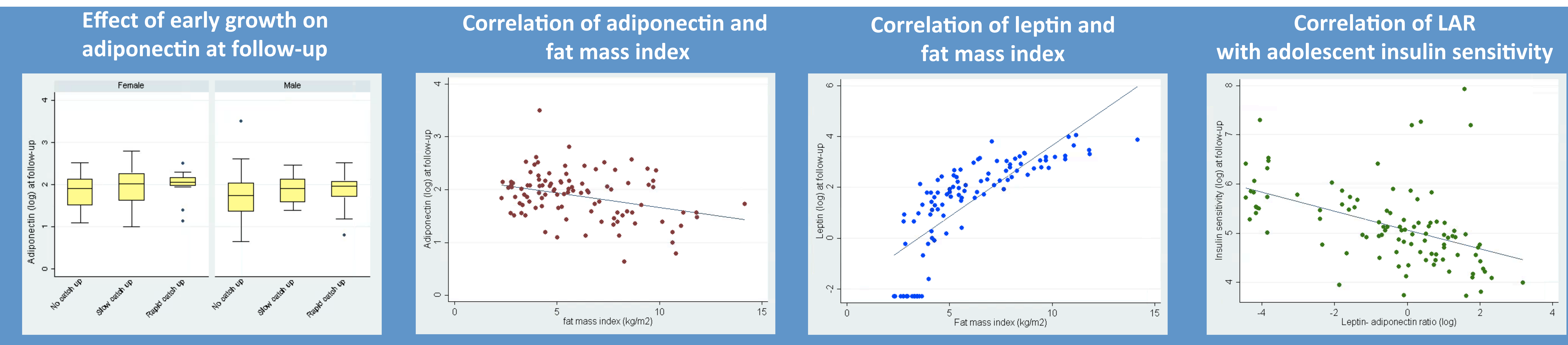

Conclusions

- We have not shown an effect of early infant growth patterns on adipokine levels at adolescent follow-up

- Contemporary body composition is a more important determinant

- The sex difference in leptin levels in the pubertal cohort may reflect sex-based differences in body fat distribution, which only evolve at puberty

- Leptin-adiponectin ratio (LAR) is a useful surrogate marker of insulin sensitivity. The LAR could replace time-consuming and invasive clamp methods and HOMA approximation when assessing insulin sensitivity in clinical research 